INRA Prod. Anim., 2007, 20 (2), 151-162

\title{
L'ingestion d'aliments par les vaches laitières et sa prévision au cours de la lactation
}

\author{
P. FAVERDIN, L. DELABY, R. DELAGARDE \\ INRA, Agrocampus, UMR1080 Production du lait, F-35590 Saint-Gilles, France
}

Courriel :Philippe.Faverdin@rennes.inra.fr

\begin{abstract}
La prévision de l'ingestion d'aliments par les vaches laitières est la clé de voûte de toute l'alimentation et de ses conséquences sur les productions, les rejets et les stocks d'aliments. Contrairement à beaucoup d'autres espèces d'élevage, tout ou partie de la ration est offerte aux vaches laitières à volonté et la mesure des quantités ingérées est difficile à réaliser. Il est donc très difficile de savoir quelles quantités consomment les vaches et le recours à des équations de prévision est particulièrement utile.
\end{abstract}

L'INRA a proposé dès 1979 un système de prévision original, le système des Unités d'Encombrement (UE) (Jarrige et al 1979). Une des qualités de ce système réside dans le fait que la prévision de l'ingestibilité des fourrages et celle de la capacité d'ingestion des animaux sont indépendantes. De ce fait, le système UE est très évolutif et peut donc être facilement amélioré sans remettre en question ni les principes du système, ni les nombreuses données de mesures d'ingestibilité sur lesquelles repose une grande partie du système. Une première révision importante avait été proposée en 1987 (Dulphy et al 1987) avec en particulier une nouvelle équation de la capacité d'ingestion des vaches laitières et un nouveau modèle de prévision de la valeur d'encombrement de l'aliment concentré (Dulphy et al 1989) utilisé dans la pratique dans le logiciel INRAtion.

Les travaux de recherches menés depuis ont permis de mieux comprendre certains mécanismes de régulation des quantités ingérées, en particulier le lien entre régulation de l'appétit et gestion des réserves corporelles. Les expérimentations réalisées ces dernières années sont venues apporter de nouvelles données pour affiner les modèles de prévision de l'ingestion d'aliments par les vaches laitières. Cet article présente les évolutions qui ont conduit à proposer un nouveau modèle, en particulier sur les liens entre ingestion et production laitière d'une part, entre ingestion et gestion des réserves corporelles d'autre part. La dernière partie traitera des évolutions du nouveau système UE pour les vaches laitières.

\section{1 / La relation complexe ingestion - production}

Depuis longtemps, les chercheurs ont observé une relation assez étroite entre la production de lait observée et les quantités ingérées. Les modèles statistiques destinés à prévoir les quantités ingérées ont toujours identifié la production laitière comme l'une des première variable prédictive. La figure 1 illustre cette relation qui, sans traduire toutes les variations de l'ingestion d'aliments, permet d'en expliquer à elle seule souvent près de $60 \%$. Elle a été obtenue sur une base de données élabo- rée à partir de 680 lactations de vaches recevant des rations à base d'ensilage de maïs et d'aliments concentrés (Leclerc et al 1993). Ce genre de relation a souvent été utilisée pour prévoir les quantités ingérées par les vaches laitières (Faverdin 1992). Cependant, en utilisant la variable production laitière qui finalement est aussi une conséquence de l'ingestion, ces équations sontelles vraiment prédictives ou sont-elles plutôt «post-dictives»?

Les vaches laitières produisent-elles plus parce qu'elles mangent plus, ou mangent-elles plus parce qu'elles produisent plus ? Cette interrogation formulée il y a plus de 40 ans traduit bien le dilemme de l'utilisation de la production laitière observée pour la prévision des quantités ingérées pour les vaches laitières. Si la production laitière dépend davantage des quantités ingérées que l'ingestion ne dépend de la production laitière, l'utilisation de la

Figure 1. Relation observée entre les quantités maximales ingérées (kg MS/j) et la production de lait maximale au sein d'une même lactation de vaches laitières primipares et multipares (Leclerc et al 1993).

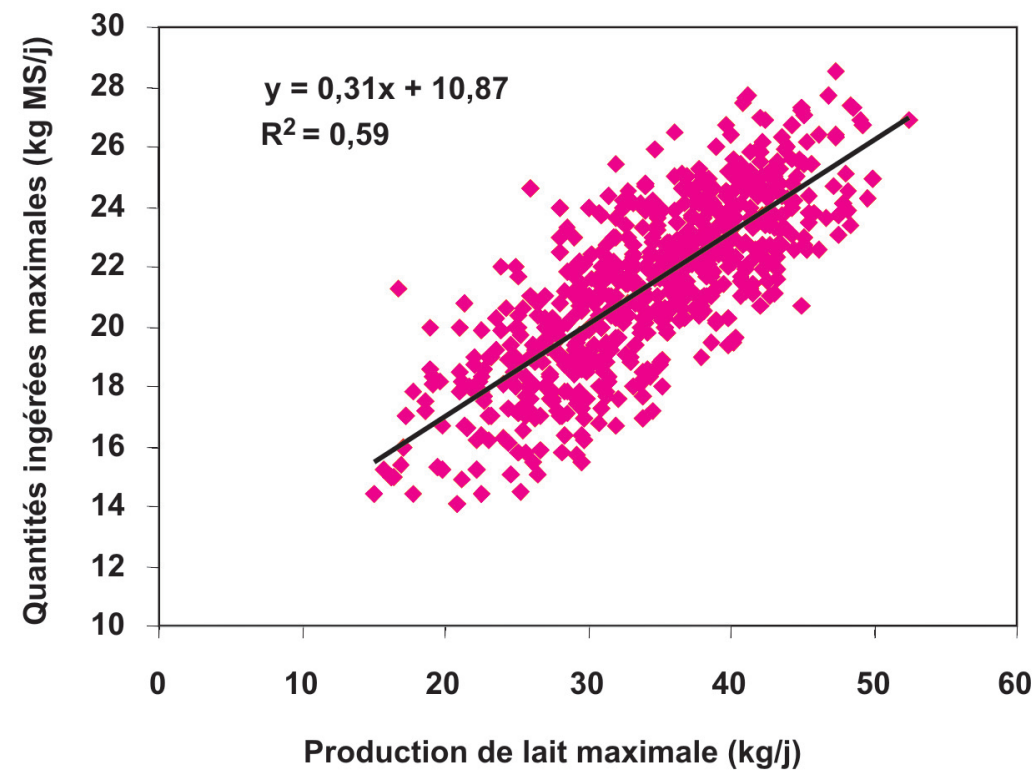


production laitière observée pour prévoir l'ingestion d'une autre ration peut conduire à des biais importants. En alimentant les vaches laitières de manière à ce qu'elles puissent à tout moment exprimer leur potentiel de production, il est vraisemblable que cette variable gardera une bonne qualité prédictive. Si par contre, la ration offerte ne permet pas à tout moment d'exprimer ce potentiel, la production laitière observée sera une variable prédictive médiocre.

\section{1 / L'augmentation d'ingestion permet d'accroître la produc- tion laitière}

L'énergie ingérée constitue généralement le principal facteur alimentaire limitant la production laitière, même si en début de lactation les réserves peuvent apporter un supplément important d'énergie. L'augmentation des quantités ingérées est souvent le premier moyen d'accroître cet apport d'énergie et donc la production laitière. Cette constatation a été faite depuis bien longtemps et des lois de réponse de l'augmentation de la production de lait à des quantités croissantes d'énergie ont été précédemment établies, pour des vaches d'un même potentiel de production (Coulon et Rémond 1991).

D'autres facteurs, comme la nutrition azotée, peuvent accroître également l'ingestion et de ce fait, augmenter l'apport d'énergie sans variation de la quantité ou de la proportion d'aliments concentrés dans la ration (Faverdin et al 2003). Ces effets peuvent parfois être spectaculaires avec des vaches fortes productrices. Dans un essai croisant 3 niveaux d'azote avec 2 niveaux d'aliments concentrés dans des rations complètes à base d'ensilage de maïs, l'augmentation d'ingestion associée à l'augmentation de production laitière est très forte (figure 2), avec environ $0,45 \mathrm{~kg}$ d'augmentation de la Matière Sèche (MS) ingérée par kg de lait supplémentaire produit (Faverdin et al 1998). De nombreux autres exemples montreraient qu'avec des lots de vaches ayant des capacités d'ingestion et de production identiques, les variations de production sous l'effet de traitements alimentaires sont d'abord liées aux variations des quantités ingérées.

Pour prévoir la capacité d'ingestion, l'utilisation de la production de lait observée avec un régime ne permettant pas une ingestion maximale d'énergie, peut conduire à une sous-estimation importante. Selon la relation observée sur la figure 2, un écart de production de près de $10 \mathrm{~kg}$ de lait observé réutilisé dans une équation de prévision de l'ingestion pourrait conduire à un écart de plus $3 \mathrm{~kg}$ de MS entre vaches ayant pourtant la même ingestion avant et après l'expérience. La production de lait observée constitue un bon indica-

Figure 2. Relation entre ingestion et production laitière observée chez des vaches laitières en début de lactation recevant des rations à base d'ensilage de maïs avec $20 \%$ ou $40 \%$ d'aliments concentrés et comportant trois teneurs croissantes de protéines (82, 97 et $112 \mathrm{~g} P D I E / U F L)$.

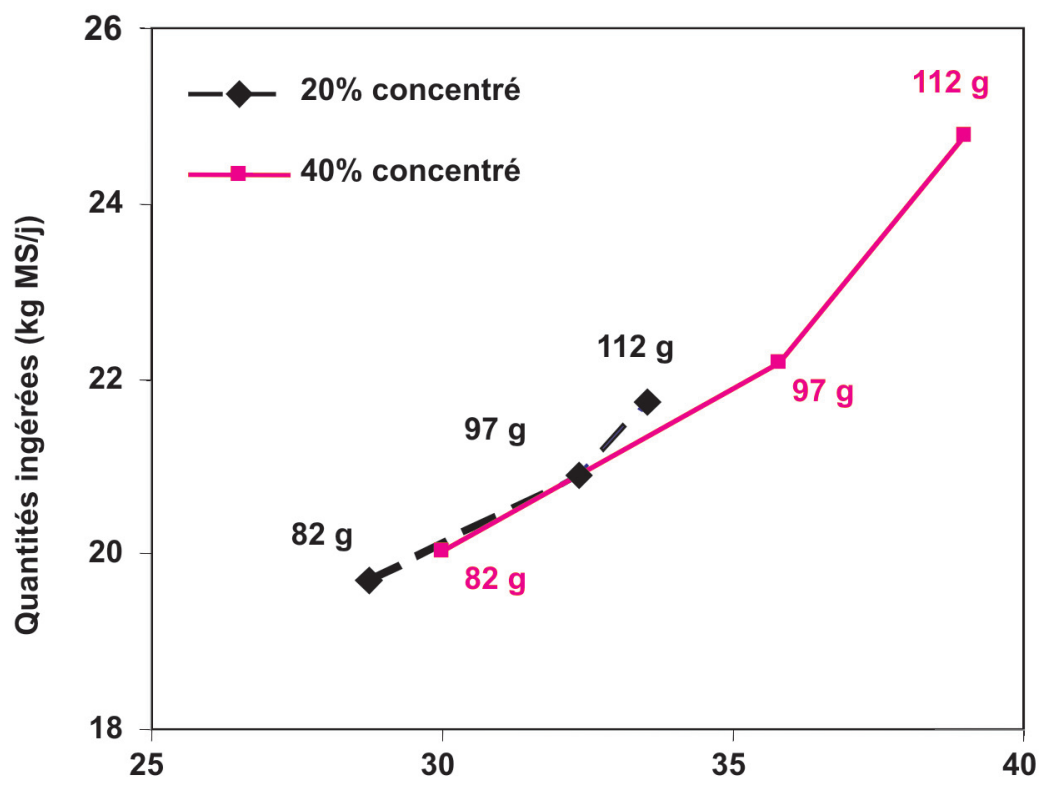

Production de lait observée $(\mathrm{kg} / \mathrm{j})$ teur des quantités qui ont été ingérées, mais elle ne permet pas de prévoir la capacité d'ingestion d'une vache laitière au cours de sa lactation.

\section{2 / L'augmentation du poten- tiel de la mamelle à produire du lait accroît également l'ingestion}

On peut définir la production de lait potentielle comme la capacité de synthèse de lait qu'aurait la mamelle à un moment donné si elle n'était pas limitée par la disponibilité en nutriments ou par un trouble sanitaire (mammite). Plusieurs grands facteurs sont susceptibles de faire varier cette production potentielle de lait d'une vache laitière. Les plus importants sont certainement le potentiel génétique, le stade de lactation, l'âge de la vache et son rang de lactation.

a) La composante génétique $d u$ potentiel de production et la capacité d'ingestion

En pratique, il est admis que plus le potentiel de production d'une vache est élevé, plus il convient de lui fournir une ration ingestible pour lui permettre de l'exprimer. Les relations positives souvent observées en France entre potentiel de production et ingestion peuvent donc s'expliquer soit par le fait que l'augmentation du potentiel stimule l'appétit, soit par le fait que les rations offertes aux vaches laitières les plus productrices sont aussi les plus ingestibles et les plus riches en énergie.

Pour estimer la composante génétique de la capacité d'ingestion, il faut en fait sélectionner des vaches selon leur index génétique «lait» et comparer l'ingestion de ces lots de vaches sur des régimes identiques. Il est par ailleurs préférable de disposer d'un régime pauvre en aliment concentré afin que l'estimation de la capacité d'ingestion ne soit pas conditionnée par la méthode d'estimation de la valeur d'encombrement de l'aliment concentré. Pour éviter toute ambiguïté, l'utilisation de l'herbe verte comme fourrage principal est souhaitable car elle constitue le fourrage de référence qui définit la valeur de l'unité d'encombrement. De plus, l'herbe verte ne présente pas l'inconvénient de contenir des quantités importantes de grain similaire à de l'aliment concentré comme dans le cas de l'ensilage de maïs.

Plusieurs essais ont été réalisés ces dernières années en Irlande (Teagasc, Moorepark) afin d'évaluer les interactions génotype $\mathrm{x}$ alimentation avec des 
Figure 3. Variation des quantités ingérées en fonction de la production de lait évoluant avec le stade de lactation du troisième mois de lactation au tarissement (Friggens et al 1998, Vérité et al non publié).

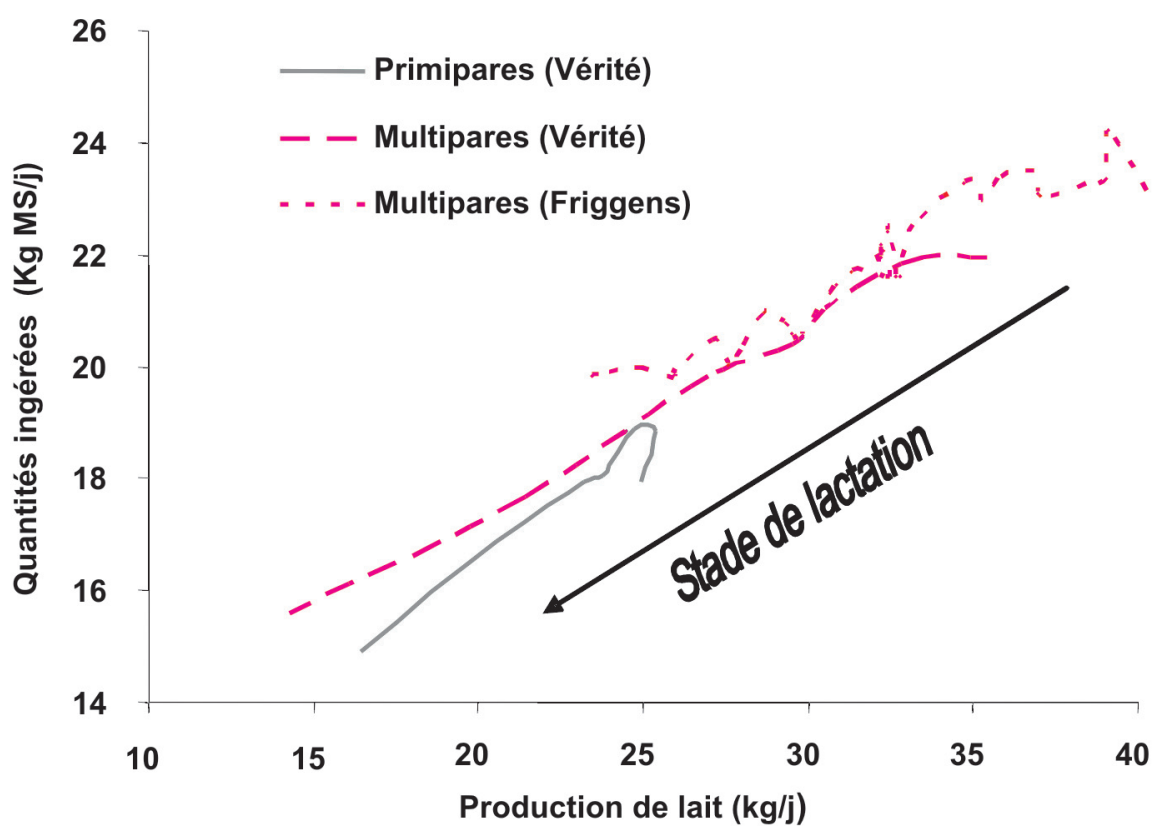

vaches laitières conduites au pâturage, en étudiant en particulier la production, l'ingestion et l'état corporel des vaches. La comparaison de différents génotypes Holstein montre clairement que la sélection génétique sur la production laitière s'accompagne d'une augmentation de la capacité d'ingestion, même au pâturage sans complémentation. Un écart de $5 \mathrm{~kg}$ de lait au pic de production lié au potentiel génétique conduit à un écart de capacité d'ingestion d'environ 1 à $1,5 \mathrm{~kg}$ de MS (Kennedy et al 2003a, Horan et al 2006). Ces résulats sont cohérents avec les équations interindividus établies entre l'ingestion d'herbe en cours d'expérience et la production laitière observée en pré-expérience qui reflète la notion de production potentielle puisque les vaches étaient alors alimentées suivant leurs besoins. Après correction du poids vif et du stade de lactation, l'augmentation d'ingestion d'herbe varie entre $0,18 \mathrm{~kg}$ et $0,25 \mathrm{~kg}$ de MS par $\mathrm{kg}$ de lait (Butler et al 2003, Peyraud et al 2004). Lorsque la production laitière observée est utilisée comme variable explicative en lieu et place de la production en préexpérience, le coefficient s'accroît considérablement pour atteindre la valeur de 0,35 à $0,50 \mathrm{~kg}$ de $\mathrm{MS}$ par $\mathrm{kg}$ de lait.

Ces travaux confirment l'hypothèse qu'au sein d'une même race, la capacité d'ingestion de fourrage est effectivement accrue lorsque l'on sélectionne des vaches ayant un potentiel de production plus élevé. Ceci n'est bien entendu pas surprenant car de nombreux auteurs ont déjà publié des corrélations génétiques fortes entre ingestion et production laitière, avec une variabilité importante des résultats allant de 0,49 à 0,95 (Hooven et al 1972, Van Arendonk et al 1991, Persaud et al 1991, Lee et al 1992). Cependant, ces corrélations génétiques n'étaient pas observées sur la consommation de fourrage seul. Pour améliorer la prévision de l'ingestion, il convient donc de prévoir la capacité d'ingestion non pas à partir de la production de lait observée qui peut être altérée par le régime en cours de distribution, mais plutôt par la production de lait potentielle de la vache.

b) Evolution de la capacité d'ingestion en fonction de la production de lait au cours de la lactation

Les variations de production laitière avec le stade de lactation sont toujours plus ou moins affectées par les variations de composition du régime, une vache recevant rarement le même régime du début à la fin de la lactation. Par ailleurs, il convient de ne pas étudier cette relation en début de lactation en raison du rôle des réserves corporelles qui sera discuté dans la partie suivante. Quelques essais ont été réalisés avec des variations assez minimes du régime au cours de la lactation (Friggens et al 1998, Vérité et al non publié). Lorsque la lactation progresse après le pic d'ingestion qui est généralement atteint au cours du $3^{\text {ème }}$ mois de lactation, la capacité d'ingestion décroît proportionnellement avec la production de lait comme l'indique la figure 3 . Cette relation présente une pente voisine de $0,27 \mathrm{~kg}$ de MS ingéré par $\mathrm{kg}$ de lait en moyenne. En apparence, la relation est assez étroite, mais elle ne permet cependant pas de dire qu'il existe un lien de cause à effet entre les deux phénomènes. Les variations des caractéristiques de la ration et de l'état corporel comme nous le verrons plus loin sont également de nature à expliquer une partie de ces variations de la capacité d'ingestion.

c) Evolution de la capacité d'ingestion en fonction de la production de lait au cours des lactations successives

Le potentiel de production s'accroît avec le numéro de lactation des vaches et ce d'autant plus que les vaches ont leur premier vêlage jeune ( 2 ans). Il est donc important d'évaluer l'évolution de la capacité d'ingestion par rapport à cette augmentation de production. On observe encore une relation de $+0,35 \mathrm{~kg}$ de MS par $\mathrm{kg}$ de lait entre l'évolution de production au cours de lactations successives et les quantités ingérées (figure 4), identique quel que soit l'âge des vaches au premier vêlage. Les données obtenues en Irlande au cours de trois lactations successives confirment également ces résultats (Kennedy et al 2003b). Comme pour le stade de lactation, il n'est possible d'évaluer l'effet intrinsèque de la production de lait potentielle qu'après l'avoir corrigé des autres facteurs associés. Dans le cas présent, il est nécessaire de prendre en compte les effets de l'âge et du format qui sont importants et vont contribuer à abaisser la valeur du coefficient lié à la production laitière.

En conclusion, ces observations confirment que la production de lait potentielle est plus pertinente que la production de lait observée pour prédire la capacité d'ingestion, même lorsque cette production potentielle ne s'exprime pas totalement. Les variations de production potentielle liées à la fois aux facteurs génétiques, mais aussi au stade de lactation et à l'âge sont positivement reliées aux variations de capacité d'ingestion. Les mécanismes impliqués ne sont pas tous clairement établis, mais une aptitude plus grande à produire s'accompagne généralement d'une aptitude à consommer accrue. Cette relation présente une pente assez faible, généralement voisine de 0,15 à $0,25 \mathrm{~kg}$ de $\mathrm{MS} / \mathrm{kg}$ de lait, et sans doute un peu plus faible après correction des 
Figure 4. Evolution des quantités ingérées au pic d'ingestion en fonction de la production maximale de lait chez des vaches laitières en lactation 1, 2 et 3 ou plus (L1, L2, L3+) vêlant à 2 ans ou à 3 ans et recevant des rations à base d'ensilage de maïs et de concentré (5,6 et 6,6 kg de MS respectivement en première lactation et au cours des lactations ultérieures). Chaque point correspond environ à une centaine de lactations (Leclerc et al 1993).

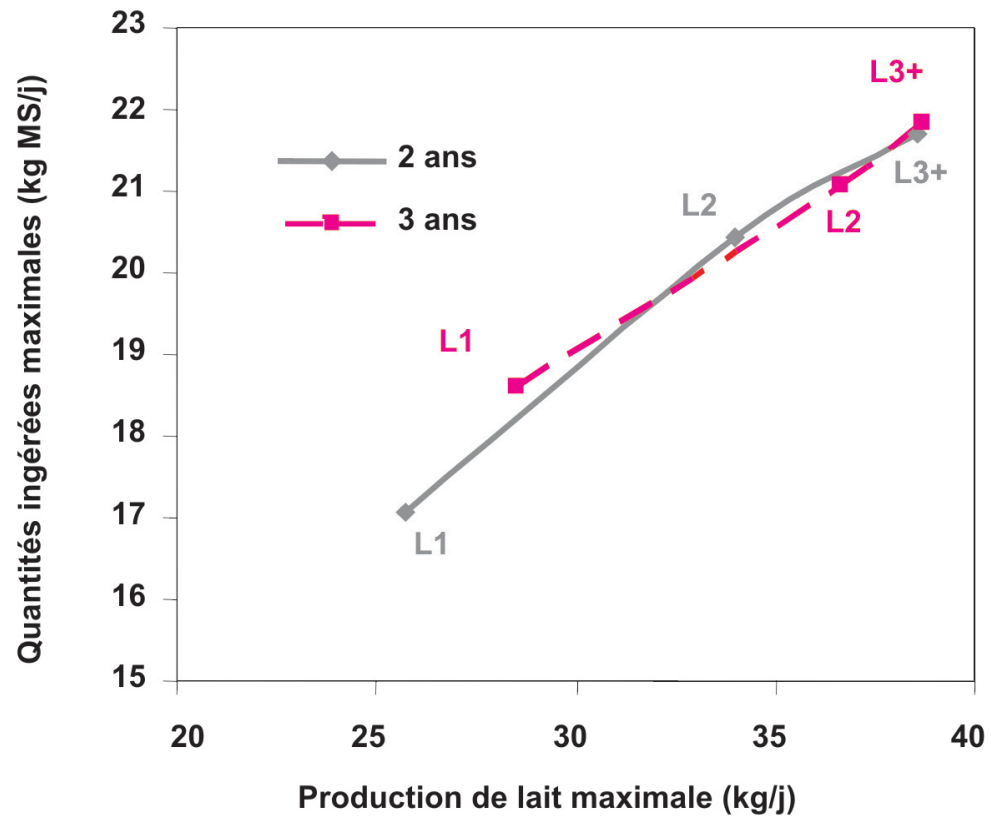

autres effets liés à l'âge, au poids, à l'état corporel ou à la gestation. Elle est globalement plus faible que la relation qui existe entre l'ingestion et la production observée qui est plus proche de 0,35 à $0,40 \mathrm{~kg}$ de $\mathrm{MS} / \mathrm{kg}$ de lait. Cette valeur est voisine de la valeur attendue sur le plan de l'équivalence énergétique $\mathrm{du}$ lait produit (proche de 0,40 à $0,45 \mathrm{~kg}$ de $\mathrm{MS} / \mathrm{kg}$ lait).

\section{2 / Le rôle des réserves cor- porelles}

\section{1 / La théorie lipostatique}

Dès 1953, Kennedy proposait la théorie lipostatique de régulation de la prise alimentaire qui suggère que le contrôle à long terme des quantités ingérées est lié à la régulation hypothalamique des réserves énergétiques corporelles. Il considère qu'après une variation d'état des réserves, la prise alimentaire est modifiée jusqu'à ce que leur point d'équilibre soit de nouveau atteint. Cela suppose l'existence d'un signal généré par les réserves adipeuses qui informe l'hypothalamus sur le niveau de ces réserves (rétrocontrôle). Par extension, cette théorie postule que les quantités ingérées sont régulées par le système nerveux central en fonction de l'adéquation entre le niveau réel des réserves adipeuses et le point d'équilibre. De façon imagée, on pourrait dire que, comme dans une entreprise, le contrôle de l'approvisionnement est fonction de l'état des stocks. tation (Roguet et Faverdin non publié)

Différence significative à $P<0,01$ **, à $P<0,05$ *

\section{2 / Restriction et suralimenta- tion}

À court et moyen terme, les animaux ajustent assez bien leur prise alimentaire à leurs besoins. Cette régulation du bilan énergétique et de la prise alimentaire est de type homéostasique, c'està-dire qu'elle permet le maintien de cet équilibre physiologique. Cette régulation semble aussi reposer sur l'existence d'un niveau normal des réserves adipeuses. Ce niveau est généralement bien récupéré après une période de sous-alimentation. Si l'on restreint volontairement un groupe de vaches laitières pendant une période de 5 semaines, elles diminuent leur production, mais mobilisent leurs réserves corporelles pour limiter cette baisse de production. Lorsque la ration leur est redistribuée à volonté, elles consomment significativement plus (1 à $2 \mathrm{~kg}$ de MS) que les vaches du lot témoin qui n'ont pas été restreintes (Roguet et Faverdin non publié, figure 5).

Chez les ruminants, comme chez de nombreuses espèces, la prise alimentaire peut être stimulée durant une longue période grâce à des rations très «palatables». Avec une ration composée de fourrages agglomérés et de concentrés, des vaches taries peuvent ingérer 2 à

3 fois plus que leurs besoins, et présen-

Figure 5. Effet d'une période de restriction alimentaire de 5 semaines sur l'évolution de l'ingestion d'une ration complète à base d'ensilage de maïs en deuxième moitié de lac-

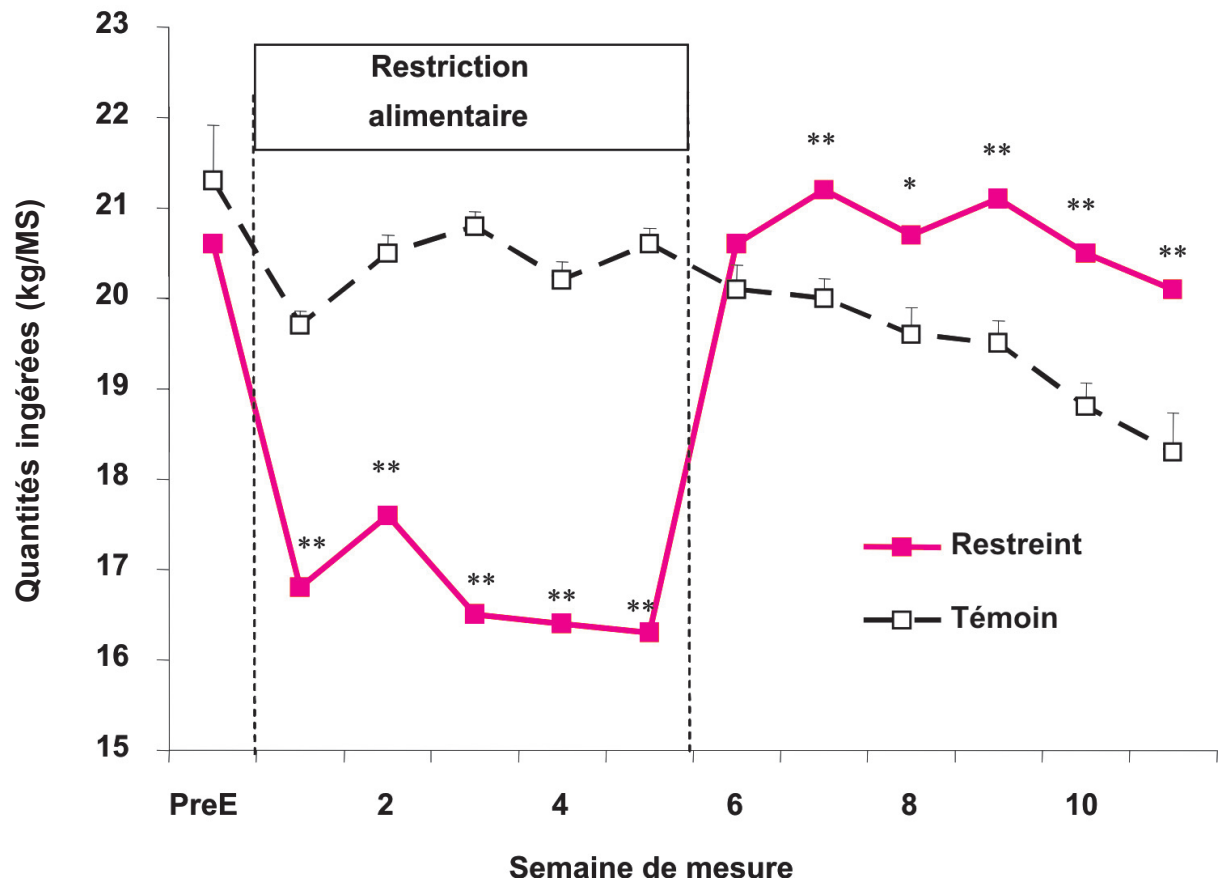


tent alors un état d'engraissement important (Faverdin et al 1992b). Cependant, on observe progressivement une baisse de l'ingestion au fur et à mesure que les réserves corporelles augmentent. Cette baisse peut s'expliquer en partie par un volume plus faible du réticulo-rumen suite à l'encombrement de l'abdomen par des réserves adipeuses, mais surtout par un effet physiologique lié à l'augmentation du niveau des réserves adipeuses et qui agit directement sur les centres de la prise alimentaire.

\section{3 / Le cycle gestation - lacta- tion}

Les femelles mammifères présentent de fortes variations de réserves adipeuses au cours de leur vie en fonction du cycle de gestation-lactation. Chez les vaches laitières, l'ingestion d'énergie augmente en début de lactation. Au cours des premières semaines après vêlage, cette augmentation n'est pas aussi rapide que celle de la production laitière et ne permet pas de couvrir les besoins d'entretien et de production (figure 6). Une partie du lait est produit à partir de la mobilisation des réserves corporelles (Chilliard et al 1987). Cette mobilisation semble dans une large mesure intrinsèque au début de lactation. En effet, si l'on augmente l'apport d'énergie en offrant une ration plus riche en aliments concentrés, l'essentiel du supplément d'énergie ingéré est utilisé par la mamelle pour produire du lait et le bilan énergétique n'est que faiblement amélioré. La mobilisation importante des réserves liée au profil hormonal du début de lactation pourrait s'expliquer par le biais d'une sensibilité accrue aux substances $\beta$-adrénergiques (Bareille et al 1997) qui affectent également l'appétit. Le délai nécessaire pour atteindre le maximum d'ingestion est un paramètre qui conditionne la durée du déficit énergétique du début de lactation. Il semble que ce délai soit influencé par la quantité de réserves adipeuses au moment du vêlage et par le niveau de production des vaches laitières. Les pertes de poids et d'état corporel en début de lactation sont d'autant plus fortes et plus longues que la note d'état corporel au vêlage et le potentiel de production sont plus élevés (Garnsworthy et Jones 1987).

Une corrélation négative entre l'état corporel au vêlage et les quantités journalières ingérées en début de lactation est souvent mise en évidence (Garnsworthy 1989, Faverdin et al 1995, Ingvartsen et al 1995). Cette relation est également retrouvée chez les ovins : les brebis maigres mangent plus que les brebis grasses en début de lactation, et la différence est d'autant plus prononcée que l'aliment est peu digestible (Cowan et al 1980). Parfois elle n'est pas observée (Boisclair et al 1986, Holter et al 1990) ce qui signifie que d'autres facteurs semblent interférer. Nous avons observé que cette sensibilité à l'excès d'engraissement sem-

Figure 6. Evolution des ingestions, des dépenses et du bilan d'énergie (UFL/j) en début de lactation de 2 groupes de vaches laitières recevant une ration complète à base d'ensilage de maïs et de $20 \%$ (tirets) ou $40 \%$ (trait continu) d'aliments concentrés pendant 14 semaines (Faverdin et al 1998). Les vaches reçoivent une même ration à $30 \%$ d'aliments concentrés avant et après la période expérimentale.

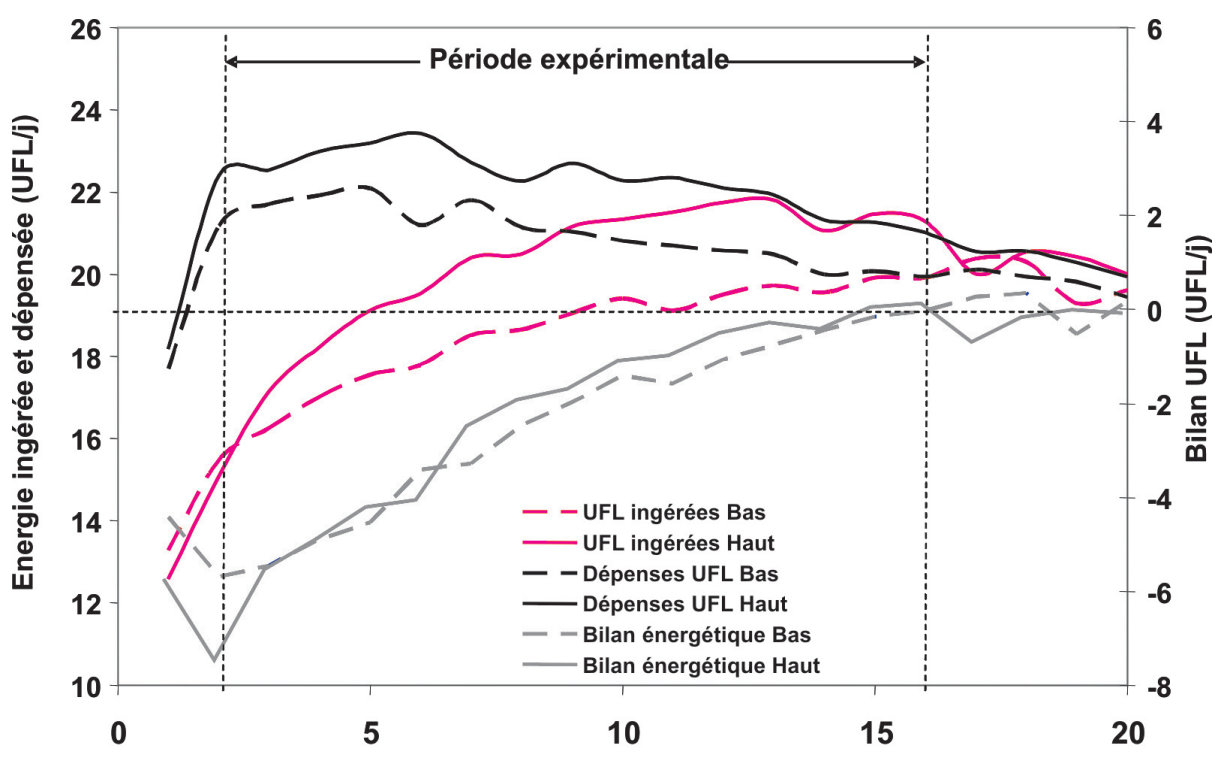

Semaine de lactation

茨

blait diminuer avec l'âge des vaches, mais les mécanismes ne sont pas connus (Leclerc et al 1993).

En revanche, à partir de 12 semaines de lactation jusqu'au vêlage suivant, l'ingestion d'énergie devient généralement supérieure aux besoins. La vache commence à reconstituer ses réserves et l'ingestion décroît alors lentement. L'ingestion des femelles laitières au cours du cycle est très liée aux variations de l'état de réserves comme l'ont suggéré Kaske et Groth (1997) chez les brebis.

La théorie lipostatique considère les réserves adipeuses comme une mémoire des écarts de la régulation de l'équilibre énergétique. Elle permet d'expliquer les variations cycliques de la prise alimentaire et des réserves adipeuses. Pour les ruminants laitiers, la prise en compte de ces mécanismes permet d'améliorer les modèles de prévision des quantités ingérées.

\section{3 / Une nouvelle équation de la capacité d'ingestion pour les vaches laitières basée sur la production de lait potentielle}

\section{1 / Rappel sur le système des unités d'encombrement}

Le système des unités d'encombrement (Jarrige et al 1979) repose sur l'idée d'attribuer une nouvelle valeur alimentaire à chaque aliment pour caractériser son aptitude à être ingérée, appelée valeur d'encombrement (UE), que l'on pourrait également appeler valeur de rassasiement. Il fait l'hypothèse que si au moins un des aliments est offert à volonté à un ruminant, la somme des valeurs d'encombrement des fourrages et des aliments concentrés pondérée des quantités de Matière Sèche Ingérées (MSI) est égale à la capacité d'ingestion de l'animal exprimée également en UE (équation 1). Les unités d'encombrement diffèrent suivant le type d'animal, celle utilisée pour les vaches laitières étant l'UEL. L'ingestion du fourrage ou du mélange offert à volonté constitue la seule inconnue de cette équation et peut donc être facilement calculée :

$$
\begin{aligned}
& C I=\sum M S I_{\text {Fourrages }} \times U E_{\text {Fourrages }} \\
& +M S I_{\text {Concentrés }} \times U E_{\text {Concentrés }} \text { Eq. } 1
\end{aligned}
$$


La Capacité d'Ingestion (CI) est prédite à partir des seules caractéristiques de l'animal, indépendamment de la ration. La valeur UE des fourrages est spécifique de chaque fourrage. Elle ne varie pas avec les caractéristiques des vaches laitières et peut-être lue dans une table de valeur d'aliments (INRA 2007). Au contraire, la valeur UE des aliments concentrés est, par construction, fonction principalement du taux de substitution entre fourrages et concentrés. Les travaux antérieurs ont montré que ce taux de substitution était principalement fonction du bilan énergétique de la vache laitière (Faverdin $e t$ al 1992a). Si la vache est en bilan positif, le taux de substitution est élevé et l'ingestion totale d'aliments augmente peu lorsque l'on apporte plus d'aliments concentrés. A l'inverse, si le bilan énergétique est négatif, le taux de substitution est faible ce qui se traduit par une augmentation de l'ingestion d'énergie lorsque l'on apporte de l'aliment concentré.

Pour prendre en compte l'évolution des connaissances sur les facteurs de variation de l'ingestion, en particulier en début de lactation, le système des UE a été récemment modifié (Faverdin et al 2006, 2007). Ces principales modifications sont explicitées dans la suite de cet article.

\section{2 / Une nouvelle notion, la pro- duction de lait potentielle}

Comme l'a montré la première partie de cet article, il existe souvent une assez bonne relation entre la capacité d'ingestion et la production de lait potentielle, quelles qu'en soient les causes de variations (potentiel génétique, stade de lactation ou numéro de lactation). D'autres modèles ont déjà pris en compte cette notion de production de lait potentielle (Sorensen et al 1992, Freer et al 1997) car elle est essentielle pour extrapoler les conséquences de la prévision de l'ingestion sur la prévision de la production de lait.

La courbe potentielle de lactation est un concept qui peut être critiqué, cette courbe potentielle pouvant être altérée par des troubles sanitaires ou des pratiques d'élevage, comme cela a été démontré avec la monotraite par exemple (Rémond et Pomiès 2005). Cependant, cette production potentielle ne semble pas affectée par une période prolongée d'alimentation de moindre qualité (Faverdin et al 1998, Vérité et Delaby 1998, Friggens et al 1998), même pendant une longue période en début de lactation (Delaby et al non publié). Pour estimer cette production potentielle, l'idée a donc été d'utiliser des modèles ajustés sur des courbes de lactation de vaches bien alimentées pendant leur lactation en paramétrant ces courbes sur la production maximale de lait observée au pic de lactation $\left(P L_{\text {MaxPot }}\right)$. Pour des raisons de simplification des calculs, le modèle retenu est celui de Wilmink (1987) modifié pour prendre en compte l'effet significatif du stade de gestation sur la production laitière comme cela avait été démontré par Bertilsson et al (1997) et déjà modélisé (Coulon et Pérochon 1998). Compte tenu des grandes différences de persistance dans la courbe de lactation des vaches primipares et multipares, deux paramétrages différents de l'équation ont été proposés :

\section{Primipares :}

$$
\begin{aligned}
P L_{\text {Pot }}= & P L_{\text {MaxPot }} \times\left[1,084-\left(0,7 \times e^{-0,46 \times S e m L}\right)\right. \\
& -(0,009 \times \operatorname{SemL}) \\
& \left.-\left(0,69 \times e^{-0,16 \times(45-S e m G}\right)\right] \quad \text { Eq. } 2
\end{aligned}
$$

Multipares :

$$
\begin{aligned}
P L_{\text {Pot }}= & P L_{\text {MaxPot }} \times\left[1,047-\left(0,69 \times e^{-0,90 \times S e m L}\right)\right. \\
& -(0,0127 \times \operatorname{SemL}) \\
- & \left.\left(0,50 \times e^{-0,12 \times(45-S e m G}\right)\right] \quad \text { Eq. } 3
\end{aligned}
$$

Avec $\operatorname{SemL}$ et $\operatorname{Sem} G$ correspondant respectivement au stade de lactation et de gestation exprimés en semaine.

Ces modèles théoriques sont difficiles à valider. Néanmoins, les données obtenues sur des troupeaux nord-américains généralement bien alimentés durant toute la lactation (Jamrozik et al 1998) permettent une évaluation sur de grands effectifs. Le tableau 1 illustre les productions observées et modélisées après avoir fixés les valeurs de $P L_{\text {MaxPot }}$ du modèle permettant d'obtenir les productions effectivement observées à 60 jours de lactation. Le modèle tend à anticiper le jour du pic de production des vaches adultes mais les données de persistance et de production globale sont très cohérentes avec les productions observées.

La seule difficulté de ce modèle consiste à renseigner la valeur de $P L_{\text {MaxPot }}$. Cette production évolue en fonction de l'âge au premier vêlage, du rang de lactation et du potentiel génétique de production de la vache. Elle augmente au cours des 3 premières lactations, puis se stabilise avant de redescendre (figure 7). Le Contrôle Laitier fournit des indicateurs de ce niveau de potentiel de production au travers de la notion de meilleur niveau d'étable exprimé. Les simulations réalisées avec le modèle indiquent qu'il existe une relation étroite entre la production potentielle cumulée sur 305 jours d'une vache et sa production potentielle maximale. On peut ainsi approcher $P L_{\text {MaxPot }}$ par le niveau d'étable, celuici correspondant en moyenne à la valeur $P L_{\text {MaxPot }}$ multipliée par 224 pour une multipare et par 259 pour une primipare. Ces méthodes d'estimation de la production de lait potentielle et de $P L_{\text {MaxPot }}$ seront à affiner à l'avenir, en particulier lorsque l'on réutilisera ces notions pour estimer la production de lait attendue en fonction des prévisions d'ingestion. En ce qui concerne la prévision de l'ingestion qui en découle, il faut néanmoins rester conscient qu'une

Tableau 1. Comparaison des données de 85414 lactations collectées par Jamrozik et al (1998) sur des vaches canadiennes aux valeurs obtenues par le modèle de production de lait potentielle.

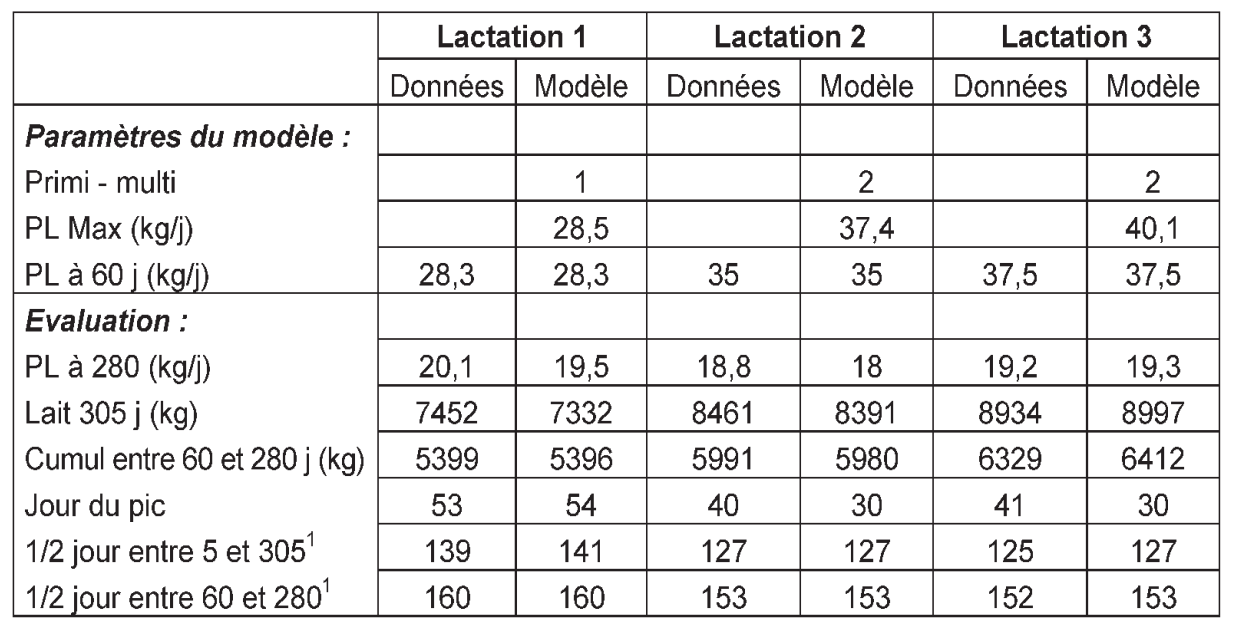

1 Le critère $1 / 2$ jour entre les jours I et $\mathrm{J}$ défini par ces auteurs correspond au jour de lactation pour lequel les vaches réalisent $50 \%$ de la production laitière cumulée entre les jours I et $\mathrm{J}$. 
Figure 7. Evolution de la production de lait maximale ( $P L$ Max) chez des vaches Holstein en fonction de l'âge au premier vêlage et du rang de lactation (Leclerc et al 1993).

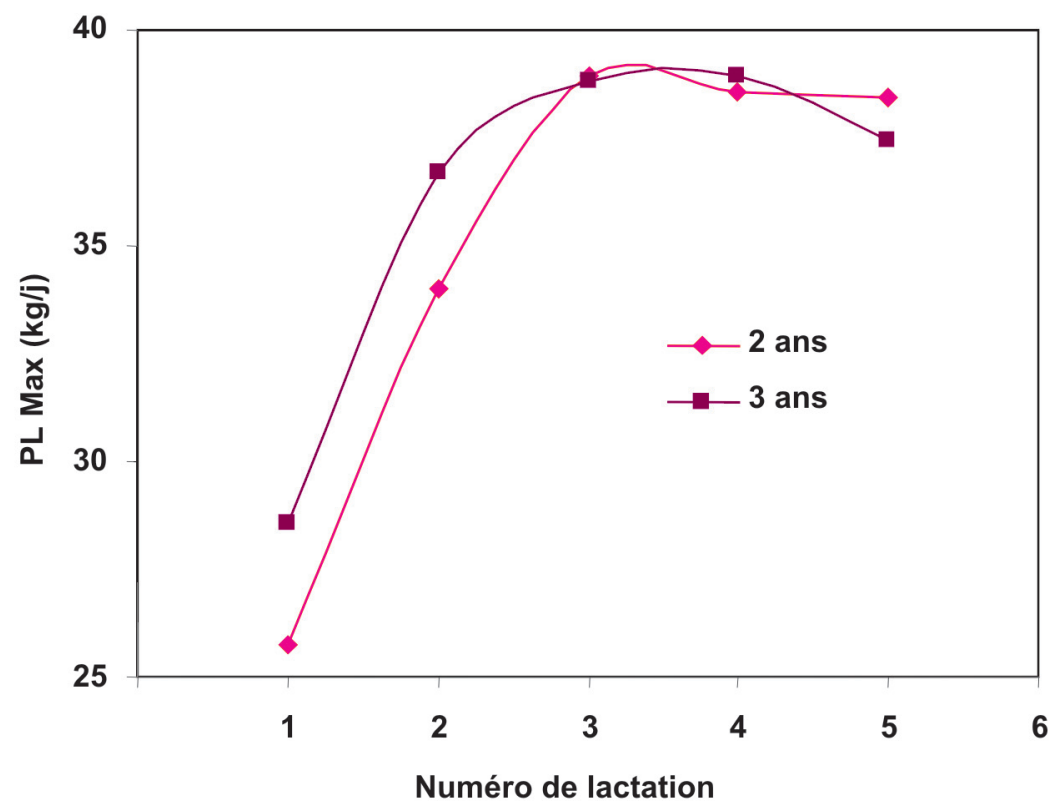

erreur de $5 \mathrm{~kg}$ de $P L_{\text {MaxPot }}$ ne conduit en général qu'à une erreur de 0,4 à $0,7 \mathrm{~kg}$ de MS de fourrage ingéré suivant les situations alimentaires et le stade de lactation.

Ce modèle de la courbe de lactation théorique des vaches laitières constitue un moyen d'évaluer la production de lait potentielle à un jour donné de lactation pour une vache d'un âge et d'un potentiel de production donnés. Il permet de prédire la capacité d'ingestion sans avoir d'information récente sur la production de la vache et de mieux prendre en compte par la suite les conséquences de l'alimentation sur la production laitière et la forme de la courbe de lait. Elle devra cependant pouvoir être améliorée lorsque les connaissances sur le déterminisme de la courbe de production potentielle seront meilleures.

\section{3 / Une nouvelle équation de la capacité d'ingestion}

L'équation précédente de capacité d'ingestion (Faverdin et al 1987) présentait plusieurs limites, en particulier pour des vaches fortes productrices ainsi qu'en début de lactation. La nouvelle formule propose un certain nombre d'évolutions de nature à mieux décrire l'ingestion de vaches laitières sans perdre le point d'ancrage de 17 UEL pour une vache adulte produisant $25 \mathrm{~kg}$ de lait et pesant $600 \mathrm{~kg}$ de poids vif.
La première évolution consiste à décrire les variations de capacité d'ingestion en fonction de la production de lait potentielle $\left(P L_{P o t}\right)$. Contrairement à la précédente équation, nous n'avons pas observé de réponse curvilinéaire entre ces deux variables. Compte tenu de toutes les analyses réalisées, le coefficient retenu est de 0,15 UEL par $\mathrm{kg}$ de lait potentiel une fois pris en compte toutes les autres variables animales (poids vif, âge et état corporel).

La seconde évolution importante concerne la prise en compte de l'effet de la Note d'Etat Corporel (NEC) sur les quantités ingérées, traduisant le rôle des réserves corporelles sur l'ingestion qui a été explicité dans la deuxième partie de cet article. En fait, cette variable est surtout utile pour prendre en compte les effets au cours du temps de la variation de note d'état d'une même vache sur l'ingestion. Elle traduit cependant moins bien l'effet sur l'ingestion des différences d'état corporel entre vaches lorsque celles-ci présentent de façon chronique des états d'engraissements faibles ou forts tout au long de leur vie. La prise en compte de la NEC conduit à accroître le coefficient du poids vif dans l'équation de prévision de la capacité d'ingestion. Cet élément vient du fait que le poids vif augmente à la fois avec l'état d'engraissement et le format de l'animal. Il confond donc en fait deux variables ayant des effets opposés sur l'ingestion. L'introduction de la note d'état dans le modèle décrit donc mieux l'effet du format de la vache sur l'ingestion. L'effet moyen de la note d'état obtenu par régression est de 1,5 UEL par point de note (échelle de 0 à 5 ). L'effet du poids vif est donc plus important que dans la précédente équation de la capacité d'ingestion qui ne comportait pas d'effet de l'état d'engraissement (1,5 au lieu de 1,0 UEL par $100 \mathrm{~kg}$ de poids vif)

$$
\begin{aligned}
& C I=\left[13,9+(0,015 \times(P V-600))+\left(0,15 \times P L_{P o t}\right)\right. \\
& +(1,5 \times(3-N E C))] \times I L \times I G \times I M \quad \text { Eq. } 4
\end{aligned}
$$

Pour mieux prévoir la dynamique d'évolution de la capacité d'ingestion au cours de la vie de la vache, trois indices ont été introduits dans le modèle. Deux d'entre eux visent à décrire les baisses de capacité d'ingestion observées autour de la mise bas, en fin de gestation et début de lactation. Le troisième permet de prendre en compte l'effet de l'âge.

L'indice de lactation (IL) traduit l'évolution, légèrement différente entre primipares et multipares, de la capacité d'ingestion au cours des premières semaines de lactation $(\operatorname{Sem} L)$ :

$$
I L=a+(1-a) \times\left(1-e^{-0,16 \times S e m L}\right) \quad \text { Eq. } 5
$$

avec $a=0,6$ pour les primipares et $a=0,7$ pour les multipares, $I L=1$ pour les vaches taries.

L'Indice de Gestation (IG) traduit la diminution de capacité d'ingestion observée au cours des dernières semaines de gestation $(\operatorname{Sem} G)$ :

$I G=0,8+0,2 \times\left(1-e^{-0,25 \times(40-\operatorname{Sem} G}\right) \quad$ Eq. 6

L'Indice de Maturité (IM) permet de décrire l'évolution de la capacité d'ingestion en fonction de l'âge de la vache (exprimé en mois). Il permet de prendre en compte le fait que la capacité d'ingestion d'une vache primipare, même corrigée du poids vif, est plus faible que celle d'une multipare, mais aussi qu'elle va évoluer en fonction de l'âge au premier vêlage :

$$
I M=-0,1+1,1 \times\left(1-e^{-0,08 \times a g e}\right) \quad \text { Eq. } 7
$$

L'équation de l'indice de maturité a été calibrée sur les données de l'essai long terme de Coulon et Ollier (1996). Après ajustement de tous les autres facteurs (poids vif, production laitière, état corporel), le rapport de la capacité d'ingestion (calculée à partir de l'équation 4 sans l'indice $I M$ ) à celle observée au cours des quatre lactations successives a été calculé et modélisé avec une fonction exponentielle (cf. figure 8) pour estimer ce nouvel indice. 
Figure 8. Evolution de l'indice de maturité en fonction de l'âge des vaches ajusté (tirets) à partir des données observées (trait continu) (Coulon et Ollier 1996).

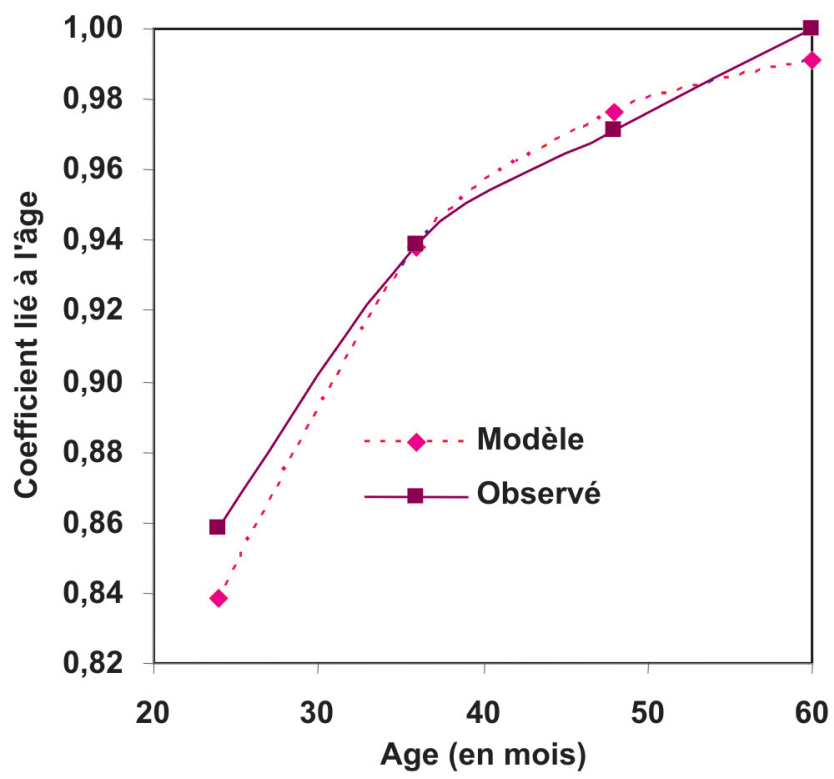

\section{4 / La prévision de l'inges- tion en début de lactation prend en compte la mobili- sation des réserves}

\section{1 / Le bilan énergétique utilisé pour le calcul de la substitution fourrages-concentrés intègre la mobilisation des réserves en début de lactation}

Les observations précédentes ont bien montré que si le taux de substitution fourrages-concentrés est bien relié au bilan énergétique de la vache en pleine lactation, ce n'est pas le cas en début de lactation. La mobilisation des réserves durant les premières semaines constitue une adaptation homéorhétique $^{1}$ de la femelle laitière (Bauman et Currie 1980), sans doute pour faire face à l'augmentation rapide des dépenses d'énergie liée à la sécrétion lactée. Elle correspond donc plus à une adaptation anticipatrice de l'organisme qu'au résultat d'un apport d'énergie insuffisant. Ceci permet de mieux comprendre pourquoi la relation entre le bilan énergétique et la substitution fourragesconcentrés est différente en début de lactation, tout en restant sensible à l'augmentation de l'apport d'aliment concentré. Un essai sur les taux de substitution en début de lactation a été réalisé pour mieux décrire cette liaison avec la situation énergétique des vaches
(Faverdin et al non publié). Un schéma expérimental spécifique a permis de comparer intra-vache l'évolution du taux de substitution pour différents niveaux d'apports d'aliments concentrés (figure 9). On observe que le taux de substitution est élevé malgré un déficit énergétique important les premières semaines de lactation, alors que le modèle de substitution précédent prévoyait des valeurs très faibles. Par contre, il existe bien une différence de taux de substitution entre les deux niveaux d'aliments concentrés malgré une différence de bilan énergétique très faible, l'essentiel de l'augmentation d'énergie ingérée ayant été exporté dans le lait.
Généralement, la valeur d'encombrement de l'aliment concentré est d'autant plus grande que la quantité d'aliment concentré ingérée est plus élevée que celle nécessaire à équilibrer le bilan énergétique de la vache. Avec la nouvelle méthode de calcul du taux de substitution, le principe général reste valable en début de lactation, mais le calcul diffère par deux éléments importants :

- le calcul des besoins énergétiques est réalisé à partir de la production laitière potentielle

- le calcul des apports énergétiques intègre un apport endogène d'énergie lié à la mobilisation des réserves considérée comme intrinsèque au début de la lactation.

On peut résumer la nouvelle méthode de calcul de ce bilan énergétique «théorique» à partir de la figure 10. Le bilan utilisé auparavant était calqué sur le calcul du bilan réel de l'animal. Le nouveau bilan théorique cherche à mesurer l'écart entre les apports d'énergie (y compris la mobilisation intrinsèque au début de la lactation) et leur utilisation potentielle, en particulier à partir des capacités de synthèses de la mamelle. Ce bilan «théorique» en début de lactation est moins déficitaire que le bilan réel, ce qui induit des valeurs d'encombrement de l'aliment concentré calculées proches des valeurs observées, tout en restant sensible au niveau d'apport d'aliment concentré. Le bilan réel varie peu avec l'apport d'aliment concentré car l'énergie consommée en plus est

Figure 9. Evolution du déficit énergétique et du taux de substitution à deux niveaux moyen d'aliments concentrés, $6 \mathrm{~kg}$ (bas) et $8 \mathrm{~kg}$ (Haut) de MS/j) en début de lactation (Faverdin et al non publié).

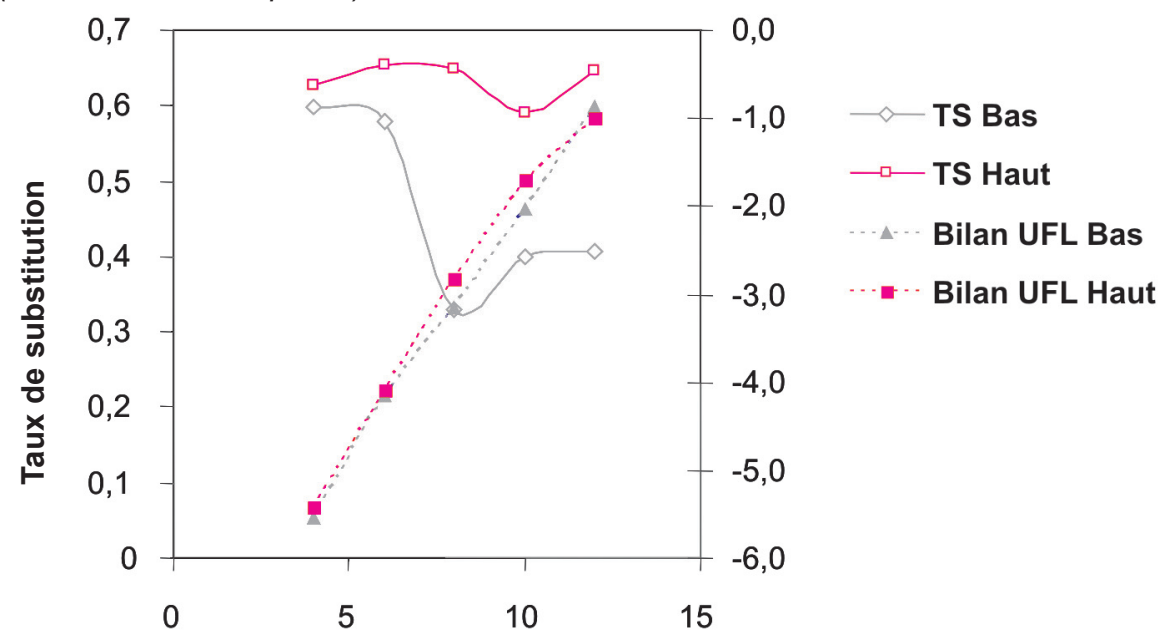

${ }^{1}$ L'homéorhèse en biologie correspond à un contrôle coordonné du métabolisme des tissus nécessaire pour permettre les changements d'états physiologiques des organismes. Cette notion est également appelée «téléophorèse» par certains auteurs (Chilliard 1993). 
Figure 10. Illustration de l'évolution de la méthode de calcul du bilan énergétique nécessaire au calcul de la valeur d'encombrement de l'aliment concentré.

\section{Ancien Bilan (INRA 1988)}

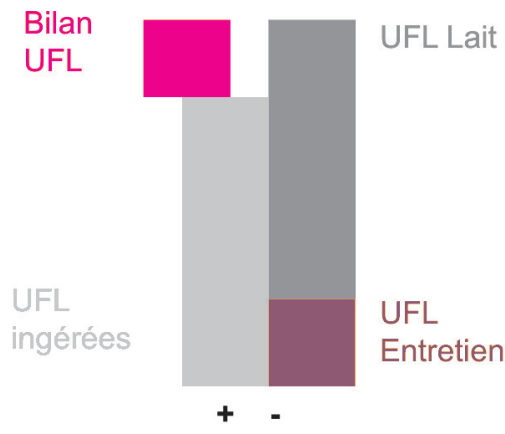

Le bilan précédent reflétait le bilan réel de l'animal. Le nouveau bilan théorique mesure l'écart entre les apports énergétiques (y compris ceux de la mobilisation intrinsèque au début de la lactation) et les besoins énergétiques prenant en compte la production potentielle de lait de la vache et non plus la production observée.

essentiellement utilisée pour accroître la production laitière. Par contre, le bilan théorique est beaucoup plus sensible car il est calculé par rapport à la production de lait théorique qui, elle, ne varie pas avec l'apport d'aliments concentré. Cette nouvelle méthode de calcul du bilan est plus sensible que le précédent aux variations d'apports énergétiques et permet une estimation plus précise de la situation énergétique de la vache.

Il reste cependant essentiel d'estimer la quantité d'énergie qui est mobilisée par une vache laitière en début de lactation. Pour cela, il a été nécessaire de développer un modèle qui quantifie la dynamique de cette mobilisation attendue des réserves en début de lactation.

\section{2 / Un modèle de la mobilisa- tion des réserves en début de lactation}

Ce modèle a été développé à partir du calcul du bilan énergétique de vaches en début de lactation dont l'ingestion et la production laitière ont été mesurées quotidiennement. Les données utilisées sont issues d'une base de données comportant 680 lactations de vaches recevant principalement des régimes à base d'ensilage de maïs (INRA, Domaine de Méjusseaume). Les valeurs de déficit énergétique observées en début de lactation sont très élevées dès la première semaine, atteignent un maximum en deuxième semaine, puis diminuent progressivement jusqu'à s'annuler au cours du deuxième ou troisième mois de lactation selon le potentiel de production des vaches.

\section{Nouveau Bilan théorique} (INRA 2007)

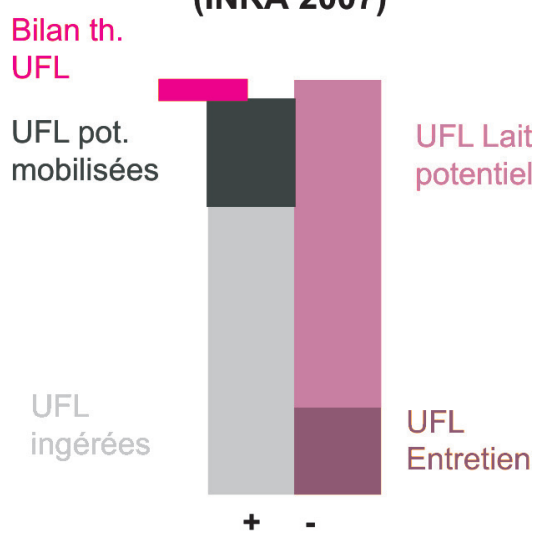

Ce modèle décrit assez bien les variations moyennes du bilan énergétique des vaches primipares et multipares en fonction de leur potentiel de production (figure 11). La mobilisation est maximale en semaine 2 où elle peut aller de 2 à 10 UFL mobilisées par jour, puis régresse rapidement au cours des semaines suivantes. La prise en compte de cette mobilisation attendue permet de calculer le bilan théorique proposé ci-dessus et de garder la cohérence du système pour la prévision de la valeur d'encombrement de l'aliment concentré. Il va permettre en particulier de bien distinguer les différences de valeurs d'encombrement de l'aliment concentré liées à des différences d'apports énergétiques par la ration, même si elles ont peu d'effet sur le bilan énergétique observé (figure 6).

\section{Conclusion}

La mobilisation varie avant tout avec le potentiel de production des vaches. Á même production en début de lactation, l'aptitude des primipares à mobiliser est supérieure à celle d'une multipare. En pratique les vaches primipares mobilisent presque autant que leurs aînées malgré une production laitière plus faible. Enfin, les vaches mobilisent d'autant plus que leurs réserves sont importantes et donc que leur note d'état au vêlage est élevée. Un point de note d'état en plus au vêlage se traduit par une mobilisation supplémentaire de 45 à 50 UFL pendant tout le début de la lactation.

Le modèle fait l'hypothèse que la vache acquière très rapidement, sous l'influence du changement hormonal du début de lactation (rapport $\mathrm{GH} /$ insuline par exemple), une très grande capacité à mobiliser ( $C$ dans le modèle), capacité qu'elle perd progressivement au cours de l'avancement de la lactation. Cette capacité à mobiliser s'accroît avec le potentiel de production $\left(P L_{P o t M a x}\right)$ et l'état des réserves corporelles $(E E)$. La traduction mathématique de ces hypothèses conduit au modèle suivant :

$$
\text { UFLmobilisées }=1-\left[C \times \frac{K 1}{K 2-K 1} \times(\exp (-K 1 \times S L)-\exp (-K 2 \times S L))\right]
$$

\section{$C$ : capacité à mobiliser}

$C=$ Apar $+0,47 \mathrm{PL}_{\text {PotMax }}+1,89 \mathrm{EE}$

avec Apar estimé à - 9,54 pour les primipares et à - 13,24 pour les multipares et EE l'état d'engraissement observé au vêlage

$K 1$ : taux d'acquisition de cette capacité, estimé à 1

$K 2$ : taux de perte de cette capacité, estimé à 0,25

$S L$ : semaine de lactation
Ces évolutions de la prévision de la capacité d'ingestion et des modes de calcul du taux de substitution vont permettre une nette amélioration des qualités prédictives du système des unités d'encombrement chez la vache laitière. Il devient en effet possible de simuler des courbes d'ingestion au cours d'un cycle complet de lactation. La possibilité de prévoir également les quantités ingérées au pâturage en fonction de son mode de conduite (Delagarde et al 2006, Faverdin et al 2007) ouvre la voie aux simulations de quantités ingérées par les vaches laitières tout au long de la lactation. La prochaine étape devra permettre de mieux simuler les conséquences des apports énergétiques et protéiques sur la production laitière et de disposer de modèles capables d'évaluer l'impact de différentes stratégies d'alimentation au cours de l'année sur les quantités de lait produites par une vache durant sa lactation ou par un troupeau tout au long de l'année. Il sera alors possible de ne plus restreindre les outils de rationnement à la question «quelle ration me permettra de couvrir les besoins d'une vache donnée ?», mais d'aller vers «quelle production avec telle ration pour telle vache ou tel 
troupeau ?». La réponse à cette dernière question est beaucoup plus complexe, mais elle est nécessaire pour aborder la très grande diversité des stratégies d'alimentation des troupeaux laitiers qui existe en pratique.

\section{Remerciements}

Les auteurs tiennent à remercier Ginette Théaud, Christine Baratte, Jean-Baptiste Coulon, Emmanuel Leclerc, Marie-Catherine LeclercRousselot, John Kennedy, Brendan Horan et Pat Dillon, pour le prêt, la mise en forme ou l'analyse de certaines données qui ont contribué à la réalisation de ce travail.
Figure 11. Evolution du déficit énergétique observé et simulé en début de lactation pour des vaches laitières primipares et multipares en fonction de leur production laitière au pic de production (Leclerc et al 1993).

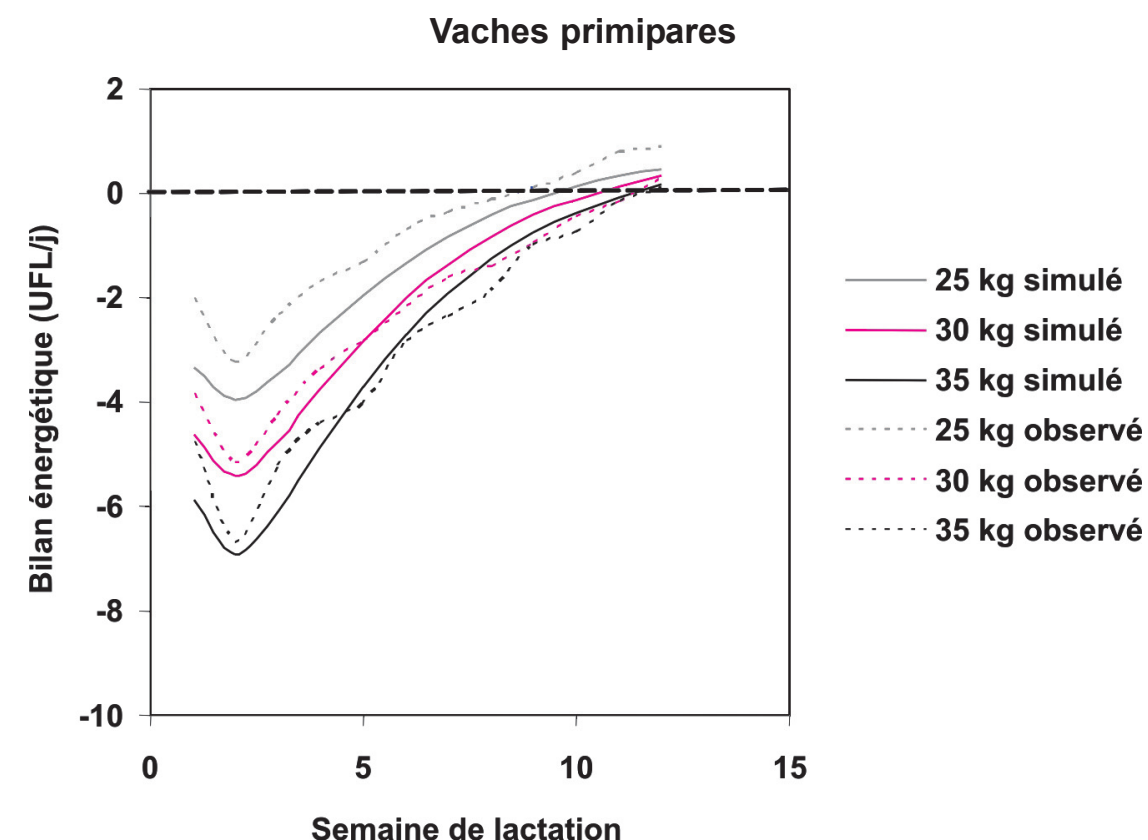

Vaches multipares

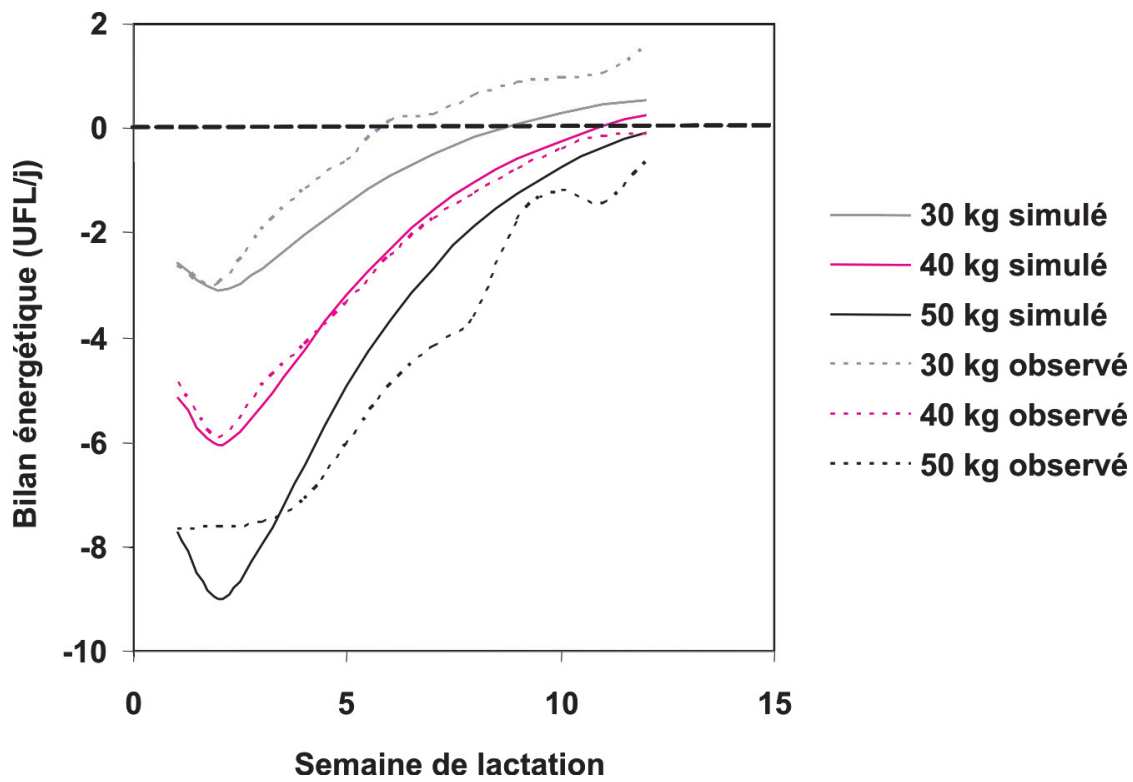

\section{Références}

Bareille N., Faverdin P., Hay M., 1997. Modification of feed intake response to a beta(2)-agonist by bovine somatotropin in lactating or dry dairy cows. J. Dairy Sci., 80, 5266.

Bauman D.E., Currie W. B., 1980. Partitioning of nutrients during pregnancy and lactation: a review of mechanisms involving homeostasis and homeorhesis. J. Dairy Sci., 63, 1514-1529.
Bertilsson J., Berglund B., Ratnayake G., Svennersten-Sjaunja K., Wiktorsson H., 1997. Optimising lactation cycles for the high-yielding dairy cow. A European perspective. Livest. Prod. Sci., 50, 5-13.

Boisclair Y., Grieve D. G., Stone J. B., Allen O. B., MacLeod G. K., 1986. Effect of prepartum energy, body condition, and sodium bicarbonate on production of cows in early-lactation. J. Dairy Sci., 69, 2636-2647.
Butler S., Stakelum G., Murphy J., Delaby L., Rath M., O'Mara F., 2003. The relationship between milk production potential and herbage intake of grazing dairy cows. Anim. Sci., 77, 343-354.

Chilliard Y., Rémond B., Agabriel J., Robelin J., Vérité R., 1987. Variations du contenu digestif et des réserves corporelles au cours du cycle gestation-lactation. Bull. Tech. CRZV Theix, 70, $117-131$. 
Chilliard Y., 1993. Adaptations métaboliques et partage des nutriments chez l'animal en lactation. In : Biologie de la lactation, Martinet J., Houdebine L.M., Head H.H. (Ed), INRA Editions, Paris, France, 431-475.

Coulon J.B., Ollier A., 1996. Residual effect of winter undernutrition applied over the first four lactations in dairy cows. Ann. Zootech., 45, 253-257.

Coulon J.B., Pérochon L., 1998. Modelling the effect of the stage of pregnancy on milk composition. Anim. Sci., 67, 413-419.

Coulon J.B., Rémond B., 1991. Variations in milk output and milk protein content in response to the level of energy supply to the dairy cow: A review. Livest. Prod. Sci., 29, 31-47.

Cowan R.T., Robinson J.J., McDonald I., Smart R., 1980. Effect of body fatness at lambing and diet in lactation on body tissue loss, feed intake and milk yield of ewes in early lactation. J. Agric. Sci., 95, 497-514.

Delagarde R., Delaby L., Faverdin P., 2006. Le calcul de ration pour vaches laitières au pâturage. Renc. Rech. Rum., 13, 89-92.

Dulphy J.P., Faverdin P., Micol D., Bocquier F., 1987. Révision du système des unités d'encombrement (UE). Bull. Tech. CRZV Theix, 70, 35-48.

Dulphy J.P., Faverdin P., Jarrige R., 1989. Feed Intake: the Fill Unit Systems. In: Ruminant nutrition-recommended allowances and feed tables, Jarrige R. (Ed.). INRA, John Libbey Eurotext, Paris, France, 61-72.

Faverdin P., 1992. Alimentation des vaches laitières : Comparaison des différentes méthodes de prédiction des quantités ingérées. INRA Prod. Anim., 5, 271-282.

Faverdin P., Hoden A., Coulon J.B., 1987. Recommandations alimentaires pour les vaches laitières. Bull. Tech. CRZV Theix, 70, 133-152.

Faverdin P., Dulphy J.P., Coulon J.B., Vérité R., Garel J.P., Rouel J., Marquis B., 1992a. Les phénomènes de substitution fourrages-concentrés chez la vache laitière. INRA Prod. Anim., 5, 127-135.

Faverdin P., Richou B., Peyraud J.L., 1992b. Effects of digestive infusions of volatile fatty acids or glucose on food intake in lactating or dry cows. Ann. Zootech., 41, 93.

Faverdin P., Leclerc E., Rousselot M.C., Troccon J.L., 1995. Evolution of intake capacity of dairy cows in early lactation. Ann. Zootech., 44, 265.

Faverdin P., Delaby L., Vérité R., Marquis B., 1998. Effet de la teneur en protéines et en aliments concentrés d'une ration complète à base d'ensilage de maïs sur l'ingestion et la production laitière de vaches laitières en début de lactation. Renc. Rech. Rum., 5, 263

Faverdin P., M'Hamed D., Rico-Gomez M., Vérité R., 2003. La nutrition azotée influence l'ingestion chez la vache laitière. INRA Prod. Anim., 16, 27-37.
Faverdin P., Delagarde R., Delaby L., 2006. Prévision de l'ingestion des vaches laitières au cours de la lactation. Renc. Rech. Rum., 13, $85-88$

Faverdin P., Delagarde R., Delaby L., Meschy F., 2007. Alimentation des bovins, ovins et caprins. Besoins des animaux. Valeurs des aliments. Tables INRA 2007. Editions Quæ, Paris, France, 23-55.

Freer M., Moore A.D., Donnelly J.R., 1997. GRAZPLAN: Decision support systems for australian grazing enterprises. II. The animal biology model for feed intake, production and reproduction and the GrazFeed DSS. Agric. Syst., 54, 77-126.

Friggens N.C., Emmans G.C., Kyriazakis I., Oldham J.D., 1998. Feed intake relative to stage of lactation for dairy cows consuming total mixed diets with a high or low ratio of concentrate to forage. J. Dairy Sci., 81, 2228-2239.

Garnsworthy P. C., 1989. The effect of energy reserves at calving on performance of dairy cows. In: Recent advances in animal nutrition 1989. W. Haresign, D.J.A. Cole (Eds). Butterworths, London, UK, 157-170.

Garnsworthy P.C., Jones G.P., 1987. The influence of body condition at calving and dietary protein supply on voluntary food intake and performance in dairy cows. Anim. Prod., 44, 347353.

Holter J.B., Slotnick M.J., Hayes H.H., Bozak C.K., Urban W.E., McGilliard M.L., 1990. Effect of prepartum dietary energy on condition score, postpartum energy, nitrogen partitions, and lactation production responses. J. Dairy Sci., 73, 3502-3511.

Hooven N.W., Miller Jr R.H., Smith J.W. 1972. Relationships among whole and part-lactation gross feed efficiency, feed consumption, and milk yield. J. Dairy Sci., 55, 1113-1122.

Horan B., Faverdin P., Delaby L., Rath M., Dillon P., 2006. The effect of strain of HolsteinFriesian dairy cow and pasture-based system on grass intake and milk production. Anim. Sci., 82 , 435-444.

Ingvartsen K.L., Danfær A., Andersen P.H., Foldager J., 1995. Prepartum feeding of dairy cattle: a review of peripartum metabolism, feed intake, production, and health T3. Proc Eur. Ass Anim. Prod., Prague, République Tchèque, 83.

INRA, 2007. Alimentation des bovins, ovins et caprins. Besoins des animaux. Valeurs des aliments. Tables INRA 2007. Editions Quæ, Versailles, France, 310p.

Jamrozik G., Jansen J., Schaeffer L.R., Liu Z., 1998. Analysis of persistency of lactation calculated from a random regression test day model. Rotorua, N. Z. Interbull Bull. 17, 64-69.

Jarrige R., Demarquilly C., Dulphy J.P., Hoden A., Robelin J., Béranger C., Geay Y., Journet M., Malterre C., Micol D., Petit M., 1979. Le système des unités d'encombrement pour les bovins. Bull. Tech. CRVZ Theix, 38, 57-79.
Kaske M., Groth A., 1997. Changes in factors affecting the rate of digesta passage during pregnancy and lactation in sheep fed on hay. Reprod. Nutr. Dev., 37, 573-588.

Kennedy G.C., 1953. The role of depot fat in the hypothalamic control of food intake in rats Proc. R. Soc. Lond., B Biol. Sci., 140, 578-592.

Kennedy J., Dillon P., Delaby L., Faverdin P. Stakelum G., Rath M., 2003a. Effect of genetic merit and concentrate supplementation on grass intake and milk production with Holstein Friesian dairy cows. J. Dairy Sci., 86, 610-621.

Kennedy J., Dillon P., O'Sullivan K., Buckley F., Rath M., 2003b. The effect of genetic merit for milk production and concentrate feeding level on the reproductive performance of Holstein-Friesian cows in a grass-based system. Anim. Sci., 76, 297-308.

Leclerc E., Rousselot M.C., Faverdin P., 1993. Capacité d'ingestion des vaches laitières en début de lactation : évolution en relation avec la production, la gestion des réserves corporelles et la génétique des animaux. Mémoire de fin d'études, Ecole Supérieure d'Agriculture d'Angers, 138p.

Lee A.J., Boichard D.A., Mac Allister A.J., Lin C.Y., Nadarajah K., Batra T.R., Roy G.L., Vesely J.A., 1992. Genetic traits of growth, feed intake and milk yield in Holstein cattle. J. Dairy. Sci., 75, 3145-3154.

Persaud P., Simm G., Hill W.G., 1991. Genetic and phenotypic parameters for yield, food intake and feed efficiency of dairy cows fed ad libitum. 1- Estimates for total lactation measures and their relationship with live-weight traits. Anim. Prod. 52, 435-444.

Peyraud J.L., Mosquer-Losada R., Delaby L., 2004. Challenges and tools to develop efficient dairy systems based on grazing: how to meet animal performance and grazing management. Grassland Science in Europe, 9, 373-384.

Rémond B., Pomiès D., 2005. Once-daily milking of dairy cows: a review of recent French experiments. Anim. Res., 6, 427-442.

Sorensen J.T., Kristensen E.S., Thysen I. 1992. A stochastic model simulating the dairy herd on a PC. Agric. Syst., 39, 177-200.

Van Arendonk J.A.M., Nieuwhof G.J., Vos H., Korver S., 1991. Genetic aspects of feed intake and feed efficiency in lactating heifers. Livest. Prod. Sci., 29, 263-275.

Vérité R., Delaby L., 1998. Conduite alimentaire et rejets azotés chez la vache laitière. Interrelations avec les performances. Renc. Rech. Rum., 5, 185-192.

Wilmink J.B.M., 1987. Adjustment of test day milk, fat and protein yield for age, season and stage of lactation. Livest. Prod. Sci., 16, 335 348 . 


\title{
Résumé
}

La qualité de la prévision de l'ingestion est essentielle dans les calculs de ration, car l'ingestion représente la principale source de variation des apports alimentaires. Cet article a pour objectif d'expliciter les évolutions récentes du système INRA de prévision de l'ingestion chez la vache laitière. La première partie présente les relations complexes qui existent entre production de lait et ingestion qui ont abouti à l'introduction de la notion de production de lait potentielle dans l'équation de prévision de la capacité d'ingestion. La seconde partie précise les relations entre ingestion et gestion des réserves corporelles et montre la nécessité d'intégrer le rôle de ces réserves dans la prévision de l'ingestion en début de lactation. Les deux dernières parties détaillent les nouvelles équations de la capacité d'ingestion des vaches laitières et du rationnement en début de lactation ; ces équations permettent une prévision plus dynamique et plus précise des quantités ingérées au cours de la lactation.

\begin{abstract}
The feed intake in dairy cows and its prediction during lactation

The accuracy of intake prediction is essential to formulate diets, because intake is the major cause of variation of nutrient supply. This article was aimed at explaining the recent evolution of the INRA system (Fill Unit System) of voluntary dry matter intake prediction in dairy cows. The first part analyses the complex relationship between milk yield and intake, which led to the introduction of the notion of potential milk yield in the equation predicting intake capacity. The second part considers the relationships between intake and regulation of body reserves and shows the importance of considering the role played by these body reserves in the prediction of intake in early lactation. The last two parts detail the new equations of intake capacity of dairy cows and of concentrate fill values in early lactation : these equations improve the intake estimation with a more dynamic and accurate prediction during lactation.
\end{abstract}

FAVERDIN P., DELABY L., DELAGARDE R., 2007. L'ingestion d'aliments par les vaches laitières et sa prévision au cours de la lactation. INRA Prod. Anim., 20, 151-162. 